\title{
Size-Dependent Bond Dissociation Enthalpies in Single-Walled Carbon Nanotubes
}

\author{
Constantinos D. Zeinalipour-Yazdi, ${ }^{\mathrm{a}, \mathrm{b}, *}$, Eriketi Z. Loizidou ${ }^{\mathrm{c}}$, Arunabhiram Chutia ${ }^{\mathrm{d}}$
}

${ }^{a}$ School of Science, University of Greenwich, Central Avenue, Chatham Maritime, Kent, ME4 4TB, UK

${ }^{b}$ Department of Chemistry, University College London, 20 Gordon Street, London, WCIH OAJ, UK

${ }^{c}$ Department of Natural Sciences, Middlesex University, Hendon Campus, The Burroughs, London, NW4 4BT, UK

${ }^{d}$ School of Chemistry, University of Lincoln, Brayford Pool, Lincoln, LN6 7TS, UK *Correspondence to: c.zeinalipouryazdi@greenwich.ac.uk

We report the bond dissociation enthalpy (BDE) and the local electronic properties of Single-Walled Carbon Nanotubes (SWCNT) using density functional theory. Our analysis shows that there is a strong size-dependence of the BDE of these SWCNTs, which is inversely proportional to the radius-squared $\left(1 / r^{2}\right)$ and the length $(1 / l)$ of SWCNT. We derive quantitative relationships from which the BDE can be calculated as a function of size and radius of the SWCNT. We find that the BDE of SWCNT outside the size-dependent region is about $480 \mathrm{~kJ}$ $\mathrm{mol}^{-1}$, which can be used for thermochemical calculations.

\section{Introduction}

Single-Walled Carbon Nanotubes (SWCNT) are used in many nanotechnology and commercial applications because of their unusual optical, electrical, mechanical and chemical properties.[1] Some of the wide range of applications include microelectronics, medicinal therapy, biosensors, gas-sensors, computing and one of their broadest use is in composite materials, to enhance their mechanical strength.[2] SWCNTs can produce materials such as nanosheets[3] and nanofibers[4,5] with very strong eleastic properties. SWCNTs filled with transition metal chains have been suggested as one-dimensional nanocables.[6] These applications rely in part on the strong elastic properties, the thermodynamic stability and inertness of SWCNTs and therefore, understanding these properties is important on a

\footnotetext{
* Corresponding author. Tel: +44 (0)208-331-9046. E-mail: c.zeinalipouryazdi@greenwich.ac.uk
} 
microscopic level. There are recent theoretical studies on structural and electronic effects of finite size zigzag or armchair carbon nanotubes of various diameters and lengths[7], their charge polarisation[8], the effect of doping[9] and the role of tube chirality on the diffusion of water.[10] There have also been studies of the properties of hybrid SWCNT with other materials, mostly carbon materials, transition metals and even confined water molecules.[11] However, there are relatively few computational studies of the thermodynamic of CNTs as a function of their radius, diameter, chirality, functionalisation and doping. To the best of our knowledge there is currently no other study of the size-dependent BDE of CNTs.

Previously, computational studies have shown that the elastic constant of zig-zag and arm-chair CNTs is smaller than that of graphene.[12] The bandgap of zig-zag $(3 n, 0)$ CNTs converges from $1.6 \mathrm{eV}$ at a length of $3 \AA$ to $0.8 \mathrm{eV}$ at a length of $9 \AA$.[12] The elastic properties (Young's, shear and bulk modulus) of functionalised CNTs have been studied with periodic density functional theory (DFT) calculations for functionalised (-NH, $\left.-\mathrm{NH}_{2},-\mathrm{CH}_{2},-\mathrm{CH}_{3},-\mathrm{OH}\right)$ organic fragments.[13] The decrease of the elastic properties is roughly proportional to the strength of the binding of the functional groups and it can be 30\% for high concentrations of adsorbates.[13] Generalized gradient approximation (GGA) based DFT calculations show that the Young's modulus of SWCNT increase as a function of their radius from $0.946 \mathrm{TPa}$ for the $\mathrm{CNT}(3,3)$ to $1.040 \mathrm{TPa}$ of the $\mathrm{CNT}(9,9)[14]$, which indicates that the C-C bond becomes stronger as the diameter of the CNT increases from $0.2 \mathrm{~nm}$ to $0.6 \mathrm{~nm}$.[14] Furthermore, hybrid exchange and correlation functional based calculations (i.e. B3LYP/6-31G) of Raman and IR spectra show that in 14 zig-zag SWCNTs there is an increasing frequency in their $\mathrm{A}_{2 \mathrm{u}}$ (bending along radial axis) and $\mathrm{E}_{\mathrm{lu}}$ (twisting along radial axis) bands, as a function of the tube diameter[15], which suggests that the elastic properties scale linearly with tube diameter.[15] The adsorption of $\mathrm{H}_{2}$ has been studied on the CNT $(10,0)$ using GGA DFT calculations, which found that the barrier for dissociative chemisorption is $79 \mathrm{kcal} \mathrm{mol}^{-1}$ and that the reaction does not happen spontaneously at $0 \mathrm{~K}$.[16] Therefore, the use of dopants such as $\mathrm{Li}^{+}$ion is necessary to use CNTs as hydrogen storage materials.[17] Energetic materials for propellants can be stored inside CNTs and are stabilised due to charge transfer from the CNT to the molecule.[18] Although these computational studies have addressed how the elastic and therefore the IR and Raman spectroscopic properties change as a function of the tube diameter and length, they have 
not given an explanation of the observed trends on the microscopic level and on the basis of the BDE of SWCNTs.

In this periodic DFT study we report the BDE and electronic properties of SWCNTs with range of lengths and diameters. In particular, we have studied the size-dependent bond dissociation enthalpy of the $\mathrm{C}-\mathrm{C}$ bond $\left(\mathrm{BDE}_{\mathrm{CC}}\right)$ and the partial-density-of-states (PDOS) of various arm-chair and zig-zag SWCNTs in the length range of $3.6 \mathrm{~nm}$ and the radius range of 1.0 $\mathrm{nm}$ in order to explain the size-dependent effect of the BDE and consequently, the elastic properties of SWCNT.

\section{Computational Methods}

\subsection{DFT calculations}

We performed periodic DFT calculations using the VASP 5.4 .1 code.[19,20] The projector augmented-wave method has been used to represent the core states. [21,22] Exchange and correlation $(\mathrm{XC})$ effects were considered within the generalized gradient approximation (GGA) using the revised Perdew-Burke-Ernzerhof (revPBE) XC functional. [23] The SWCNT, were optimised inside an orthorhombic cell with a $20 \AA$ vacuum gap between the CNTs and an axial vacuum gap of $15 \AA$ as shown in Fig. 1 .

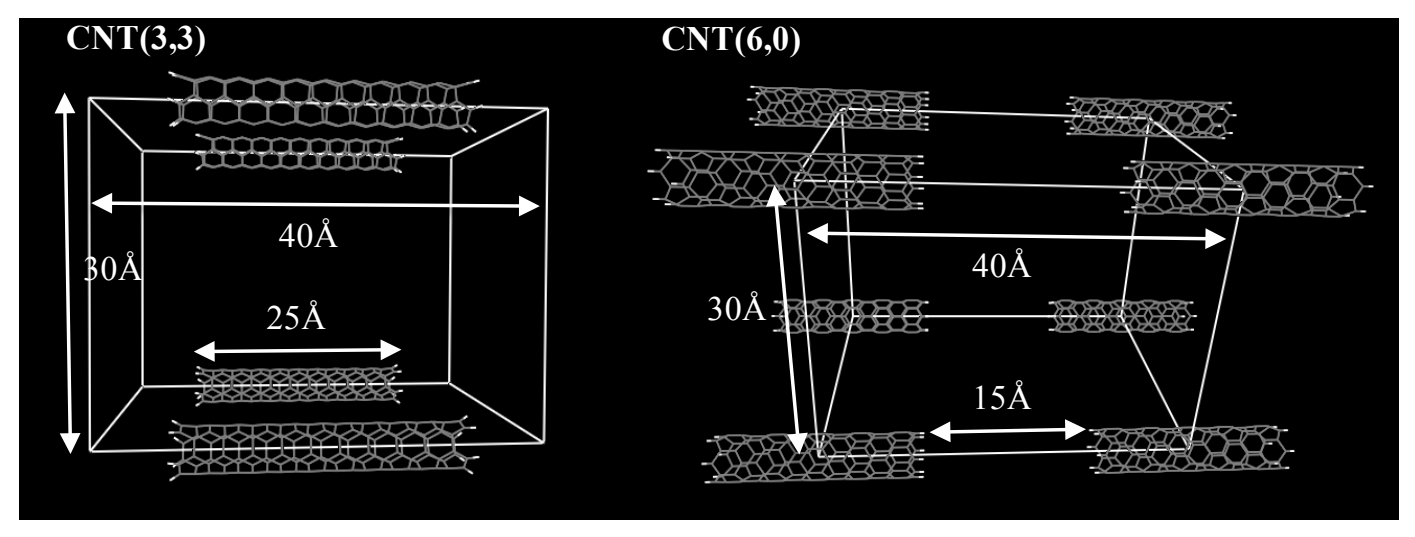

Fig. 1. The periodic unit cell of $\operatorname{SWCNT}(3,3)$ and $\operatorname{SWCNT}(6,0)$. The SWCNT length is $25 \AA$. For the $30 \AA$ and $35 \AA$ in length SWCNT, the unit cell width was $45 \AA$ and $50 \AA$, respectively.

We have used a $\Gamma$-point[24] centered 2x2x2 Monkhorst-Pack grid for all calculations but the convergence of the energy was tested also with a 3x3 $33 \mathrm{MP}$ grid which gave energies to within $0.0001 \mathrm{eV}$. The coordinates of the SWCNT were generated in Nanotube Modeler[25] and 
then rotated and translated to the highest space group using Materials Studio.[26] The corresponding point and space groups of each SWCNT are tabulated in Table 1. The dangling bonds in all SWCNT were saturated with one hydrogen atom per carbon atom. The cut-off energy for the energy of the planewaves was $600 \mathrm{eV}$. Geometry optimizations were performed with a residual force threshold on each atom of $0.01 \mathrm{eV} \AA^{-1}$ using the conjugate-gradient algorithm. The convergence criterion for electronic relaxation was $10^{-4} \mathrm{eV}$. The initial charge density was obtained by superposition of atomic charges. Dispersion corrections were included via the zero-damping DFT-D3 correction method of Grimme as implemented in VASP.[27]

\subsection{Calculation of bond dissociation enthalpies}

The change in enthalpy for the bond formation of an SWCNT from its atoms was calculated based on the following equation,

$$
\Delta H \cong E_{S W C N T}-n_{C} E_{C}-n_{H} E_{H}+n_{C} Z P V_{C}
$$

, where $n_{C}$ and $n_{H}$ is the number of $\mathrm{C}$ and $\mathrm{H}$ atoms, respectively. $E_{S W C N T}, E_{C}$ and $E_{H}$ are the total energies of the SWCNT, of an isolated carbon atom in its triplet state $(-1.626 \mathrm{eV})$ and a hydrogen atom in its doublet state $(-1.178 \mathrm{eV})$ and the zero-point vibrational energy per carbon atom $\left(Z P V_{C}\right)$ derived from a full electron B3LYP/STO-3G(d,f) calculation of the $Z P V_{C}$ of circum-circum-coronene $\left(\mathrm{C}_{96} \mathrm{H}_{12}\right)$ given by,

$$
Z P V_{C} \cong \frac{Z P V\left(C_{96} H_{24}\right)}{n_{C}}
$$

The enthalpy of atomization of the SWCNT was calculated based on the following relationship,

$$
\Delta H_{\text {atom }}=n_{C C} B D E_{C C}+n_{C H} B D E_{C H}
$$

, where $B D E_{C H}$ and $B D E_{C C}$ are the average bond-dissociation energies of the $\mathrm{C}-\mathrm{H}$ bond and C-C bond, respectively. The change in enthalpy for the bond formation of the SWCNT is equal to the negative atomization enthalpy,

$$
\Delta \mathrm{H} \cong-\Delta \mathrm{H}_{\text {atom }}
$$

Therefore, after the combination of equations 1, 3 and 4, the average BDE per C-C bond in an arbitrary SWCNT becomes,

$$
B D E_{C C} \cong \frac{1}{n_{C C}}\left[-E_{S W C N T}+n_{C} E_{C}+n_{H} E_{H}-n_{C} Z P V_{C}-n_{C H} B D E_{C H}\right]
$$


The $B D E_{C H}$ was estimated based on the energy required to dissociate a hydrogen atom from coronene $\left(R=C_{24} H_{12}\right)$ forming the corresponding radical $\left(R^{*}=C_{24} H_{11}^{*}\right)$ given by,

$$
B D E_{C H}=E_{R H}-E_{R} \cdot E_{H}+Z P V_{C H}
$$

For the average $B D E_{C H}$ of coronene we have used the value $454 \mathrm{~kJ} \mathrm{~mol}^{-1}$, which was calculated at B3YP/cc-pVDZ(5d,7f) level of theory.

The ZPV of C-H was calculated with the following equation,

$$
Z P V_{C H}=Z P V_{R H}-Z P V_{R} \text {. }
$$

, where $Z P V_{R H}$ and $Z P V_{R^{*}}$ are the zero-point vibrational energies of coronene and coronene radical in its triplet state calculated at B3LYP/cc-pVDZ(5d,7f), respectively.[28]

\section{Results and Discussion}

\subsection{Size-dependent properties of SWCNT as a function of their length}

To the best of our knowledge, the trends of the bond dissociation enthapy per C-C bond $\left(\mathrm{BDE}_{\mathrm{CC}}\right)$ as a function of SWCNT length and radius, have not been previously reported. We have used equations 1-7 to calculate this thermodynamic property for various arm-chair and zigzag SWCNT in which the dangling bonds were saturated with $\mathrm{H}$-atoms. These results are tabulated in table 1 along with the chirality, point group (P.G.), symmetry group (S.G.), length (l), radius ( $\mathrm{r}$ ), number of $\mathrm{C}-\mathrm{C}$ bonds $\left(\mathrm{n}_{\mathrm{CC}}\right)$ and the number of $\mathrm{C}$ and $\mathrm{H}$ atoms $\left(\mathrm{n}_{\mathrm{C}}, \mathrm{n}_{\mathrm{H}}\right)$.

Table 1. Chirality, point group (P.G.), symmetry group (S.G.), length (1), radius (r), number of $\mathrm{C}-\mathrm{C}$ bonds $\left(\mathrm{n}_{\mathrm{CC}}\right)$, the number of $\mathrm{C}\left(\mathrm{n}_{\mathrm{C}}\right)$ and $\mathrm{H}\left(\mathrm{n}_{\mathrm{H}}\right)$ atoms, length (1), radius $(\mathrm{r})$ and the bond dissociation enthalpy per $\mathrm{C}-\mathrm{C}$ bond is $\left(\mathrm{BDE}_{\mathrm{CC}}\right)$ of the various armchair and zig-zag SWCNTs. Also shown the average BDE of C-C and C=C. [29] 


\begin{tabular}{ccccccccc}
\hline Chirality & P. G. & S. G. & $\begin{array}{c}\mathbf{I} \\
(\AA)\end{array}$ & $\begin{array}{c}\mathbf{r} \\
(\AA)\end{array}$ & $\mathbf{n}_{\mathbf{C C}}$ & $\mathbf{n}_{\mathbf{C}}$ & $\mathbf{n}_{\mathbf{H}}$ & $\mathbf{B D E}_{\mathbf{C C}}$ \\
& & & & & & $(\mathrm{kJ} / \mathrm{mol})$ \\
\hline$(6,0)$ & $\mathrm{D}_{6 \mathrm{~h}}$ & PMMM & 26.480 & 4.861 & 210 & 144 & 12 & 456.2 \\
$(7,0)$ & $\mathrm{D}_{7 \mathrm{~h}}$ & PMM2 & 26.499 & 5.484 & 245 & 168 & 14 & 462.1 \\
$(8,0)$ & $\mathrm{D}_{8 \mathrm{~h}}$ & P4/MMM & 26.494 & 6.403 & 280 & 192 & 16 & 465.8 \\
$(9,0)$ & $\mathrm{D}_{9 \mathrm{~h}}$ & PMM2 & 26.524 & 7.061 & 315 & 216 & 18 & 468.7 \\
$(10,0)$ & $\mathrm{D}_{10 \mathrm{~h}}$ & PMMM & 26.509 & 7.956 & 350 & 240 & 20 & 470.6 \\
$(3,3)$ & $\mathrm{D}_{3 \mathrm{~h}}$ & $\mathrm{PM}$ & 26.352 & 4.009 & 183 & 126 & 12 & 450.4 \\
$(4,4)$ & $\mathrm{D}_{4 \mathrm{~h}}$ & $\mathrm{P} 4 / \mathrm{M}$ & 26.627 & 5.551 & 244 & 168 & 16 & 463.4 \\
$(5,5)$ & $\mathrm{D}_{5 \mathrm{~h}}$ & $\mathrm{PM}$ & 26.651 & 6.860 & 305 & 210 & 20 & 469.4 \\
$(6,6)$ & $\mathrm{D}_{6 \mathrm{~h}}$ & $\mathrm{P} 2 / \mathrm{M}$ & 26.644 & 8.254 & 366 & 252 & 24 & 472.6 \\
$(7,7)$ & $\mathrm{D}_{7 \mathrm{~h}}$ & $\mathrm{PM}$ & 26.627 & 9.605 & 427 & 294 & 28 & 474.6 \\
$(3,3)$ & $\mathrm{D}_{3 \mathrm{~h}}$ & $\mathrm{PM}$ & 11.585 & 4.009 & 79 & 54 & 12 & 428.4 \\
$(3,3)$ & $\mathrm{D}_{3 \mathrm{~h}}$ & $\mathrm{PM}$ & 16.507 & 4.009 & 114 & 78 & 12 & 439.4 \\
$(3,3)$ & $\mathrm{D}_{3 \mathrm{~h}}$ & $\mathrm{PM}$ & 21.430 & 4.009 & 149 & 102 & 12 & 445.2 \\
$(3,3)$ & $\mathrm{D}_{3 \mathrm{~h}}$ & $\mathrm{PM}$ & 26.352 & 4.009 & 183 & 126 & 12 & 450.4 \\
$(3,3)$ & $\mathrm{D}_{3 \mathrm{~h}}$ & $\mathrm{PM}$ & 31.275 & 4.009 & 218 & 150 & 12 & 451.3 \\
$(3,3)$ & $\mathrm{D}_{3 \mathrm{~h}}$ & $\mathrm{PM}$ & 36.197 & 4.009 & 253 & 174 & 12 & 452.8 \\
$(6,0)$ & $\mathrm{D}_{6 \mathrm{~h}}$ & $\mathrm{P} 2 / \mathrm{M}$ & 9.052 & 4.700 & 70 & 48 & 12 & 425.3 \\
$(6,0)$ & $\mathrm{D}_{6 \mathrm{~h}}$ & $\mathrm{P} 2 / \mathrm{M}$ & 13.315 & 4.700 & 105 & 72 & 12 & 441.1 \\
$(6,0)$ & $\mathrm{D}_{6 \mathrm{~h}}$ & $\mathrm{P} 2 / \mathrm{M}$ & 17.578 & 4.700 & 140 & 96 & 12 & 448.9 \\
$(6,0)$ & $\mathrm{D}_{6 \mathrm{~h}}$ & $\mathrm{P} 2 / \mathrm{M}$ & 21.841 & 4.700 & 175 & 120 & 12 & 453.5 \\
$\mathrm{C}-\mathrm{C}$ & $\mathrm{n} / \mathrm{a}$ & $\mathrm{n} / \mathrm{a}$ & $\mathrm{n} / \mathrm{a}$ & $\mathrm{n} / \mathrm{a}$ & $\mathrm{n} / \mathrm{a}$ & $\mathrm{n} / \mathrm{a}$ & $\mathrm{n} / \mathrm{a}$ & 377 \\
$\mathrm{C}=\mathrm{C}$ & $\mathrm{n} / \mathrm{a}$ & $\mathrm{n} / \mathrm{a}$ & $\mathrm{n} / \mathrm{a}$ & $\mathrm{n} / \mathrm{a}$ & $\mathrm{n} / \mathrm{a}$ & $\mathrm{n} / \mathrm{a}$ & $\mathrm{n} / \mathrm{a}$ & 728 \\
\hline & & & & & & & &
\end{tabular}

In order to study the $\mathrm{BDE}_{\mathrm{CC}}$ as a function of SWCNT length we have choosen the zig-zag SWCNT(6,0) and arm-chair SWCNT(3,3), which have about the same radius, $4.861 \AA$ and 4.009 $\AA$, respectively, and had a relatively small number of carbon atoms so that longer tube lengths could be simulated. These SWCNT have relatively small diameter and therefore systems in which these have lengths as $4 \mathrm{~nm}$ could be studied. The bond dissociation enthalpy per C-C bond is proportional to 1/length of the CNT as this is shown in Fig. 2. This means that as the length of the SWCNT increases the $\mathrm{BDE}_{\mathrm{CC}}$ becomes larger. For SWCNT that have longer lengths, there should be a convergence of the $\mathrm{BDE}_{\mathrm{CC}}$ to a constant value, which however cannot be observed for $\mathrm{CNT}$ lengths $<4 \mathrm{~nm}$. In contrast we observe that the $\mathrm{BDE}_{\mathrm{CC}}$ changes between $425-454 \mathrm{~kJ}$ $\mathrm{mol}^{-1}$, corresponds to $6.3 \%$. We suggest that changes in the elastic properties of CNTs for 
lengths $<4 \mathrm{~nm}$ could be as high as $6.3 \%$, which means that size-dependent elastic properties have to be considered to accurately access such properties. This noticeable variation of the $\mathrm{BDE}_{\mathrm{CC}}$ also suggests size-dependence of the thermodynamic properties for SWCNT that have lengths below $4 \mathrm{~nm}$. This size-dependence of the properties should be observable in measurements of the elastic properties of SWCNT, such as Young's modulus, the tensile strength and the bending modulus, all of which are dependent on the bond strength of the $\mathrm{C}-\mathrm{C}$ atoms within the carbon nanotube framework. Therefore, longer SWCNTs have in principle stronger mechanical properties such as Young's modulus and bending modulus and tensile strength as we have previously shown that there is linear correlation between the Young's modulus and the binding energy in graphene nanoribbons.[30] SWCNT are known to have the ability to withstand changes in length when under lengthwise tension or compression, which is a direct result of their high Young's modulus. The broad range of $\mathrm{BDE}_{\mathrm{CC}}$ found in this study indicates that also there should be a broad range for the elastic properties of SWCNTs and MWCNTs. Analysis of the stress-strain curves of individual Multi-Walled Carbon Nanotubes (MWCNT) reveals Young's modulus of the outermost SWCNT in the range of 270 to $950 \mathrm{GPa}$ measured with a "nanostressing stage" located within a scanning electron microscope.[31] The tensile strength of this layer varied from 11 to $63 \mathrm{GPa}$.[31] The wide range of Young's modulus and tensile strength in MWCNT is evident from the broad range of $\mathrm{BDE}_{\mathrm{CC}}$ which were found to be in the range 428 $\mathrm{kJ} \mathrm{mol}^{-1}-453 \mathrm{~kJ} \mathrm{~mol}^{-1}$ and $425 \mathrm{~kJ} \mathrm{~mol}^{-1}-454 \mathrm{~kJ} \mathrm{~mol}^{-1}$ for the $\mathrm{CNT}(3,3)$ and $\operatorname{CNT}(6,0)$, respectively. These CNTs had lengths that ranged between 1 to $4 \mathrm{~nm}$ and larger values for the $\mathrm{BDE}_{\mathrm{CC}}$ are expected for longer SWCNT. By setting the length in the equation of the line in Fig. 1 to infinity we get a value of $463 \mathrm{~kJ} \mathrm{~mol}^{-1}$ and $480 \mathrm{~kJ} \mathrm{~mol}^{-1}$ for the $\operatorname{CNT}(3,3)$ and $\operatorname{CNT}(6,0)$, respectively. These BDE values are not identical as there is a $0.86 \AA$ radius difference between the two SWCNTs, and we show in the next section that there is a considerable radius dependence of the BDE of CNTs. Currently BDE are not available in the literature, however we can compare to BDE of organic molecules. In particular, we find that the BDE of SWCNT outside the sizedependent region is about $480 \mathrm{~kJ} \mathrm{~mol}^{-1}$ which is higher than that of the $\mathrm{C}-\mathrm{C}$ bond, $377 \mathrm{~kJ} \mathrm{~mol}^{-1}$ and lower than that of the $\mathrm{C}=\mathrm{C}$ bond, $728 \mathrm{~kJ} \mathrm{~mol}^{-1}$, suggesting a partial double bond in SWCNTs.[29] 


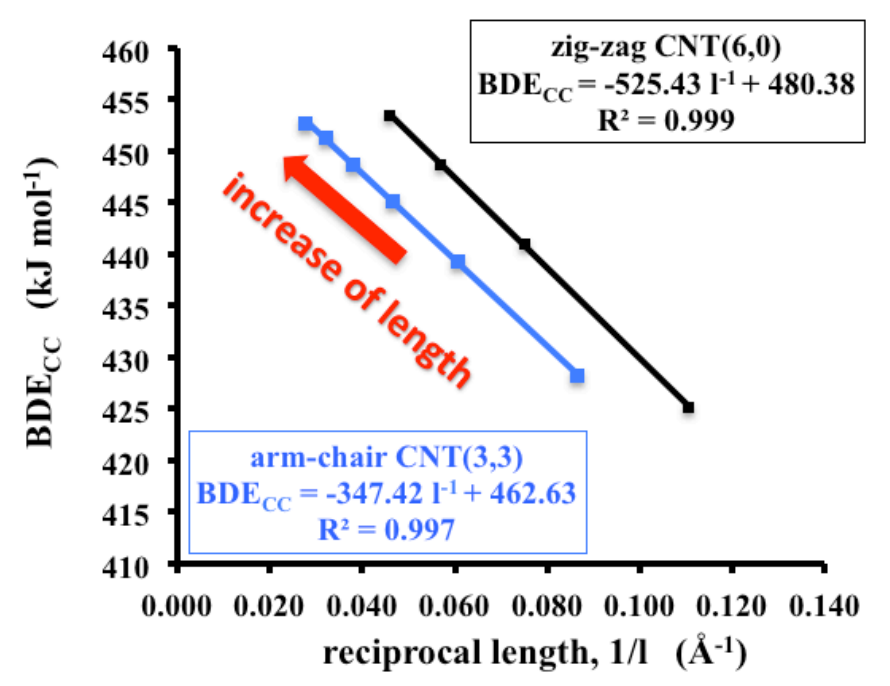

Fig. 2. Bond dissociation enthalpy per $\mathrm{C}-\mathrm{C}$ bond as a function of the reciprocal CNT length. The length of the CNT(6,0) and CNT(3,3) ranged between $9.052 \AA-21.841 \AA$ and $11.585 \AA-36.197$ $\AA$, respectively.

In Fig. 3 the converging trend of the $\mathrm{BDE}_{\mathrm{CC}}$ can be seen. It is intriguing that even for 3 $\mathrm{nm}$ long CNTs the $\mathrm{BDE}_{\mathrm{CC}}$ has yet to converge, which suggests that there is a size-dependence that governs this thermodynamic property. The fit to the data points is an equation of the form $\mathrm{BDE}_{\mathrm{CC}}=\mathrm{A}-\mathrm{B} / \mathrm{l}$, where $\mathrm{A}$ and $\mathrm{B}$ are constants. This equation yielded residual-sum-of-squares (RSS) of 0.13 and 0.06 , which indicates a good fit of the calculated data-points. By setting $1=\infty$ for the fit equation the $\mathrm{BDE}_{\mathrm{CC}}$ of an infinite long CNT becomes 465 and $474 \mathrm{~kJ} \mathrm{~mol}^{-1}$ for $\operatorname{SWCNT}(3,3)$ and SWCNT $(6,0)$, respectively, values that are close to the results obtained earlier from the linear extrapolation. From Fig. 3 we estimate the length at which the $\mathrm{BDE}_{\mathrm{CC}}$ converges, which is $8.3 \mathrm{~nm}$ and $8.7 \mathrm{~nm}$ for $\operatorname{SWCNT}(3,3)$ and $\operatorname{SWCNT}(6,0)$, respectively. This suggests that in SWCNT and MWCNT changes of the mechanical properties are expected for lengths $<9 \mathrm{~nm}$ and that only CNTs with dimensions $>9 \mathrm{~nm}$ should have isotropic thermodynamic and elastic properties (i.e constant as a function of size). This is quite important in the correct design of nano-, nano-sensors and nano-mechanical resonators that utilise CNTs. 


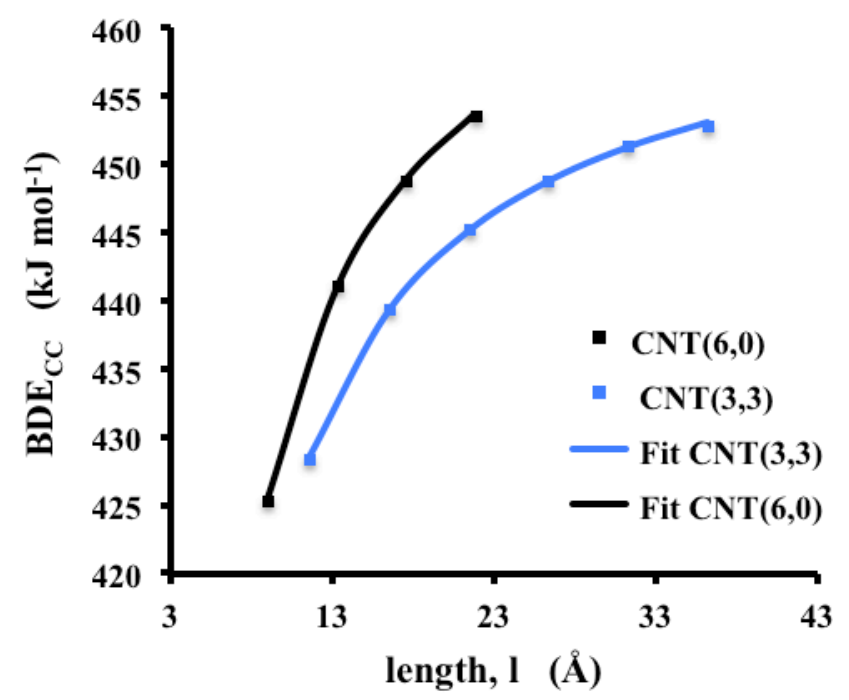

Fig. 3. Bond dissociation enthalpy per C-C bond as a function of the CNT length. The length of the $\mathrm{CNT}(6,0)$ and $\mathrm{CNT}(3,3)$ ranged between $9.052 \AA-21.841 \AA$ and $11.585 \AA-36.197 \AA$, respectively.

\subsection{Size-dependent properties of SWCNT as a function of radius}

The radius of a CNT is a significant geometric parameter for CNTs as it determines the curvature of the hexagonal carbon sheet that is rolled into the tubular structure. The larger the radius or diameters of a CNT the flatter its surface. For CNTs with radius greater than $10 \mathrm{~nm}$ electronic properties similar to graphene would be expected. In Fig. 4 the $\mathrm{BDE}_{\mathrm{CC}}$ is plotted as a function of $\mathbf{1} /$ radius $^{2}$ of various arm-chair (i.e. CNT(n,n) where $\left.\mathrm{n}=3,4,5,6,7\right)$ and zig-zag (i.e. $\mathrm{CNT}(\mathrm{m}, 0)$ where $\mathrm{m}=6,7,8,9,10)$ chirality $\mathrm{CNTs}$, respectively, with various lengths (i.e. 9.0 $36.2 \AA$ ) and radii (i.e. $4.2-9.6 \AA$ ). The excellent fitting of the $1 /$ radius $^{2}$ to the BDE can be explained by similar fitting of the strain energies that has been previously been found in first principle calculations of the elastic properties of CNTs.[32] Similar 1/radius ${ }^{2}$ dependance of the strain energy was found in carbo nano-toroids by Goddard and co-worker.[33] The arm-chair CNTs have higher $\mathrm{BDE}_{\mathrm{CC}}$ than the zig-zag $\mathrm{CNT}$ for lengths below $4 \mathrm{~nm}$ and radii under $1 \mathrm{~nm}$. This suggests that arm-chair CNT have a larger Young's modulus, tensile strength, and bending modulus than zig-zag SWCNTs when their length is below $4 \mathrm{~nm}$ and their radius less than $1 \mathrm{~nm}$.

The lines in Fig. 4 are perfectly linear based on the $\mathrm{R}^{2}=0.999$ and $\mathrm{R}^{2}=0.994$ for armchair and zig-zag, respectively. For an infinitely long SWCNT the arm-chair CNT(n,n) and zigzag $\mathrm{CNT}(\mathrm{m}, 0)$ we find that the $\mathrm{BDE}_{\mathrm{CC}}$ becomes $480 \mathrm{~kJ} \mathrm{~mol}^{-1}$ and $479 \mathrm{~kJ} \mathrm{~mol}^{-1}$, which means that 
on the basis of radius both arm-chair and zig-zag have the same $\mathrm{BDE}_{\mathrm{CC}}$ (taking into consideration the numerical limitations of the calculations) when their radius is large. Furthermore, at small radii (radius $=1 \mathrm{~nm}$ ) the $\mathrm{BDE}_{\mathrm{CC}}$ of arm-chair CNTs is similar to the $\mathrm{BDE}_{\mathrm{CC}}$ of zig-zag CNTs to within $2 \mathrm{~kJ} \mathrm{~mol}^{-1}$. Therefore, the $\mathrm{BDE}_{\mathrm{CC}}$ of both arm-chair and zigzag CNTs is the same as a function of their radius or curvature..

Also in Fig. 4 we observe that the $\mathrm{BDE}_{\mathrm{CC}}$ becomes greater as the radius of the CNTs increases, which means that the carbon nanotube becomes thermodynamically more stable. We explain this increase of the thermodynamic stability via PDOS plots in section 2 which show that the overlap of the $p$ orbitals of the $\pi$-system is greater when the curvature is smaller (i.e larger radii).

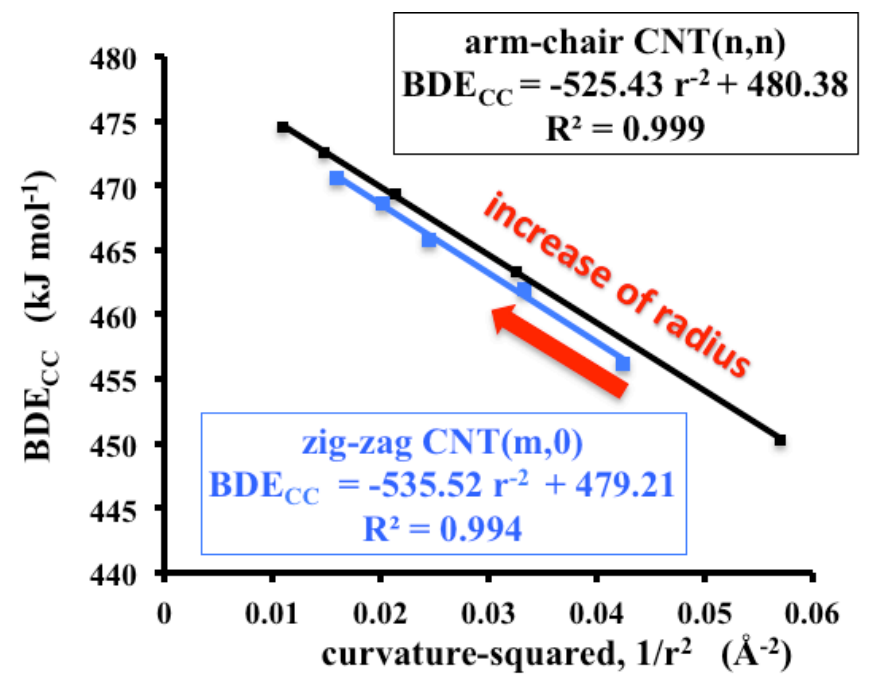

Fig. 4. Bond dissociation enthalpy per C-C bond as a function of $1 / \mathrm{r}^{2}$ for arm-chair $\mathrm{CNT}(\mathrm{n}, \mathrm{n})$ and zig-zag $\operatorname{CNT}(m, 0)$, where $\mathrm{n}=3,4,5,6,7$ and $\mathrm{m}=6,7,8,9,10$. The radius of the $\mathrm{CNT}(\mathrm{n}, \mathrm{n})$ and $\mathrm{CNT}(\mathrm{m}, 0)$ ranged between $4.191 \AA-9.605 \AA$ and $4.861 \AA-7.956 \AA$, respectively.

In Fig. 5 we have plotted the $\mathrm{BDE}_{\mathrm{CC}}$ as a function of the SWCNT radius in order to show the converging trend of this thermodynamic property. Similar to the conclusions reached from Fig. 4, we observe that a radius of $10 \AA$ is not sufficient to observe convergence of the $\mathrm{BDE}_{\mathrm{CC}}$. By fitting these curves to a function of the form $\mathrm{BDE}_{\mathrm{CC}}=\mathrm{A}-\mathrm{B} / \mathrm{r}^{2}$ we have seen that at infinite radius, the $\mathrm{BDE}_{\mathrm{CC}}$ of the arm-chair and zig-zag SWCNT become $480.2 \mathrm{~kJ} \mathrm{~mol}^{-1}$ and $479.2 \mathrm{~kJ}$ $\mathrm{mol}^{-1}$, respectively, which are identical within the accuraccy of DFT calculations $\left(\sim 1-2 \mathrm{~kJ} \mathrm{~mol}^{-1}\right)$ 
but we also find that the $\mathrm{BDE}_{\mathrm{CC}}$ becomes constant, to within $0.1 \mathrm{~kJ} \mathrm{~mol}^{-1}$, for SWCNT, with radii greater than $7.3 \mathrm{~nm}$. Therefore, bundles of SWCNT that have radii greater than $7 \mathrm{~nm}$ will have isotropic elastic properties which is an important design feature in the use of CNTs in various applications.

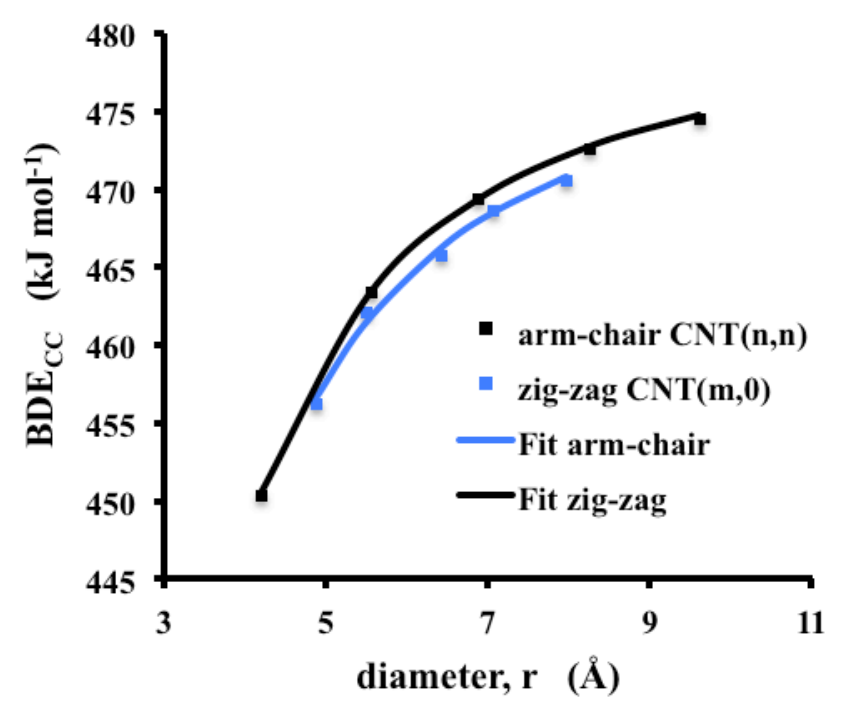

Fig. 5. Bond dissociation enthalpy per $\mathrm{C}-\mathrm{C}$ bond as a function of the radius for arm-chair CNT(n,n) and zig-zag CNT(m,0), where $n=3,4,5,6,7$ and $m=6,7,8,9,10$. The radius of the $\mathrm{CNT}(\mathrm{n}, \mathrm{n})$ and $\mathrm{CNT}(\mathrm{m}, 0)$ ranged between $4.191 \AA-9.605 \AA$ and $4.861 \AA$ - $7.956 \AA$, respectively.

The trends in the $\mathrm{BDE}_{\mathrm{CC}}$ of CNTs as a function of their diameter can be explained considering that there are certain energy requirements to bend a planar graphene sheet and roll it into a nanotube. We have previously studied what the bending modulus is in graphene nanoribbons and found that energy is required to bend a graphene nanoribbon (GNR), which suggests that carbon materials with curved surfaces have higher energies than flat GNR.[30] The bending energy is considerably smaller than the shearing and compression energy as we have previously shown for GNR of varying sizes.[30] When the GNR is bent there is less overlap between the $\mathrm{C} p_{z}$ orbitals primarily, which causes the $\mathrm{BDE}_{\mathrm{CC}}$ to decrease as the curvature of the $\mathrm{CNT}$ increases. Furthermore, the largest $\mathrm{BDE}_{\mathrm{CC}}$ should be observed in flat graphene sheets.[28,34] This means that in MWCNT the outermost SWCNT will always have the largest elastic properties, such as Young's modulus, tensile strength, and bending modulus. 
(a)

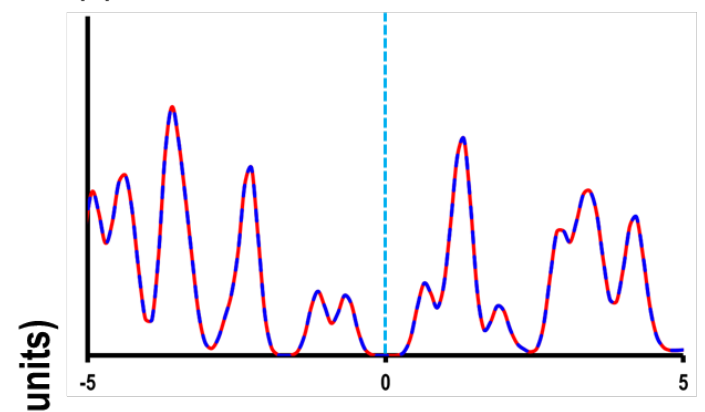

$\frac{2}{\pi}(b)$

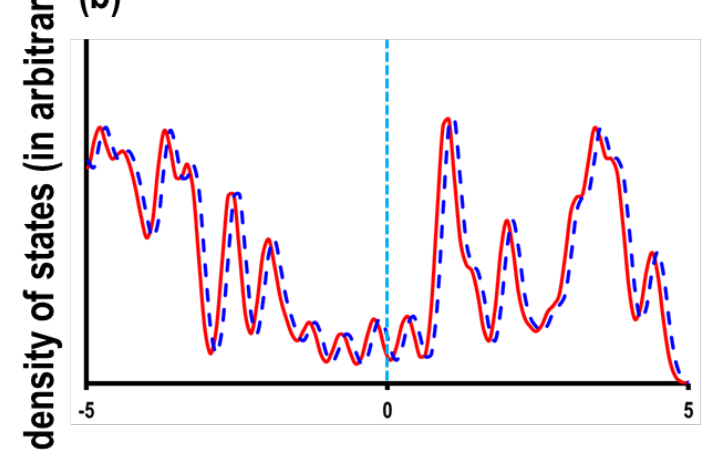

$\frac{\frac{\pi}{0}}{\frac{\pi}{\pi}}$

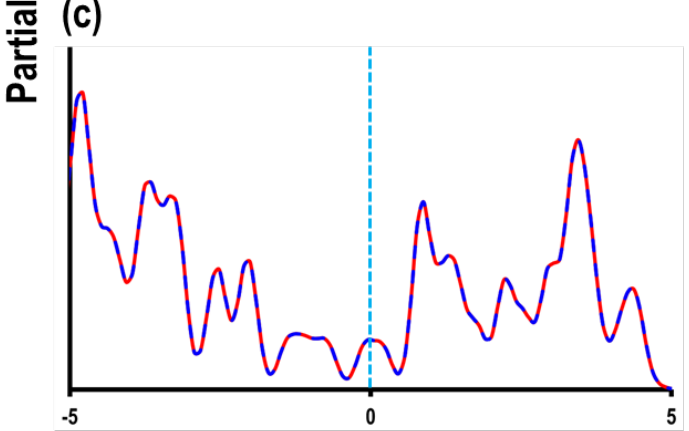

(d)

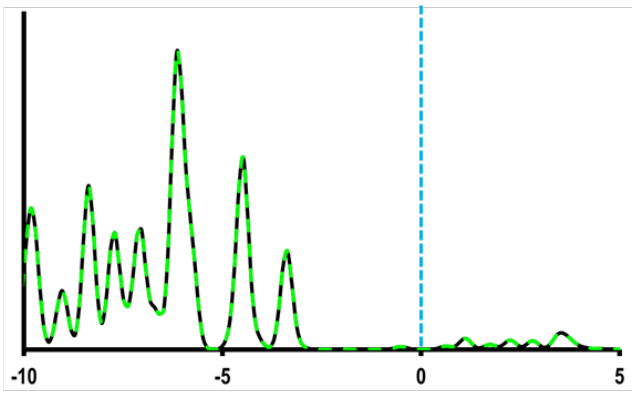

(e)

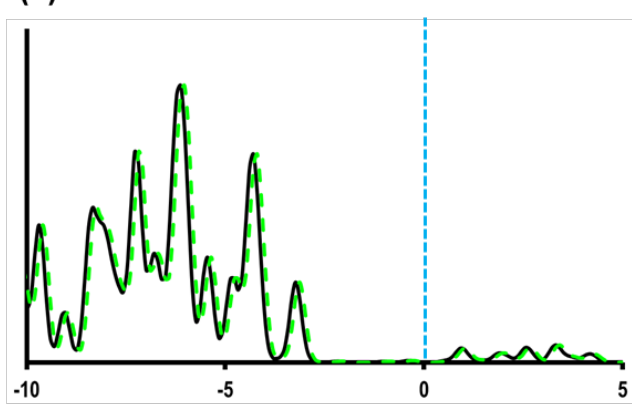

(f)

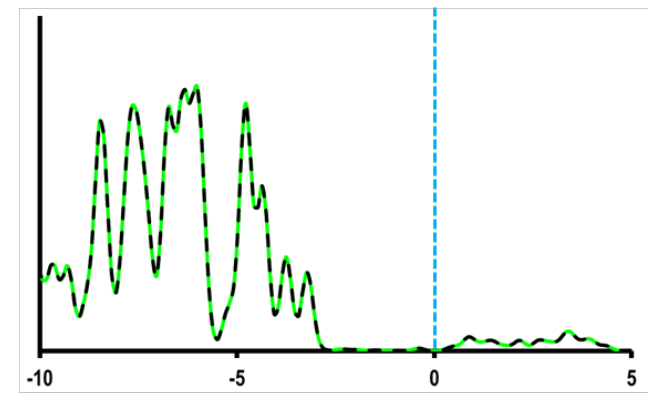

Energy (eV)

Fig. 6. Partial-density-of-states (PDOS) for the two carbon atoms in the centre of the CNT $(3,3)$ with lengths of (a) $10 \AA$, (b) $20 \AA$ and (c) $30 \AA$. The solid red (is the first carbon atom) and dotted blue (is the second carbon atom) lines, represents the summation of the contribution from $\mathrm{C}\left(p_{x}, p_{y}\right.$ and $\left.p_{z}\right)$ orbitals of the two carbon atoms from the center of the CNT under consideration. The PDOS of the $\mathrm{p}_{\mathrm{z}}$ orbitals (solid black line for the first carbon atom and the 
green dotted line for the second carbon atom) of the $\operatorname{CNT}(3,3)$ with length of (d) $10 \AA$, (e) $20 \AA$ and (f) $30 \AA$. The vertical dotted line at zero represents the Fermi energy $\left(E_{F}\right)$.

Density-of-states (DOS) have been previously used to explain the closing of the bandgap in SWCNTs as their dimensions increase.[12] However, in order to understand the sizedependent BDE trends of SWCNTs we have considered the Partial density of states (PDOS), which shows how the overlap between $p$ electrons changes as a function of the SWCNT dimension. First, in order to understand the increase in the $\mathrm{BDE}_{\mathrm{CC}}$ with increasing length of the SWCNT we consider the PDOS for the CNT(3,3) with length of $10 \AA, 20 \AA$ and $30 \AA$ (see Fig. 6 (a-c)). For these plots, we choose two carbon atoms from the center of the SWCNTs. The summation of $p_{x}, p_{y}$, and $p_{z}$ contributions around the Fermi energy $\left(\mathrm{E}_{\mathrm{F}}\right)$ show that with increasing length, the gap between the highest occupied and the lowest unoccupied states near the $\mathrm{E}_{\mathrm{F}}$ decrease (Fig. $6(\mathrm{a}-\mathrm{c})$ ). We also observe that for the $\operatorname{SWCNT}(3,3)$ with $30 \AA$ length there are $\mathrm{C} p$ states at the $\mathrm{E}_{\mathrm{F}}$, meaning that this $\operatorname{SWCNT}$ is conducting while $\operatorname{SWCNT}(3,3)$ with a length of $10 \AA$, and $20 \AA$ are semiconducting. To clarify the chemical bonding in these two carbon atoms we analyse the overlapping between their $\mathrm{C} p$ orbitals. As shown in Fig. $6(\mathrm{~d}-\mathrm{f})$, the $\mathrm{C} p_{z}$ orbitals, as expected, are completely overlapped with each other but with the increasing length of the SWCNT $(3,3)$ these overlapping $\mathrm{C} p_{z}$ orbital signatures become broader. In particular, the area under the PDOS plots from $-5.5 \mathrm{eV}$ to $-2.7 \mathrm{eV}$ was found to be $0.55 \mathrm{eV}, 0.57 \mathrm{eV}$ and 0.63 $\mathrm{eV}$ for the $10 \AA, 20 \AA$ and $30 \AA \operatorname{SWCNT}(3,3)$, respectively. An increased overlap between $p_{z}$ orbitals means that the $\mathrm{C}-\mathrm{C}$ bonds become stronger as the length of the $\mathrm{CNT}$ increases. This also explains the increase in $\mathrm{BDE}_{\mathrm{CC}}$ with the increase in the SWCNT lengths. This observation has significant applications to it as it suggests that CNT-composite materials that have the same $\operatorname{mass}(\mathrm{CNT}) /$ mass (composite) the one with the lengthier CNTs will have in principle larger Young's modulus, tensile strength and bending modulus. 
(a)

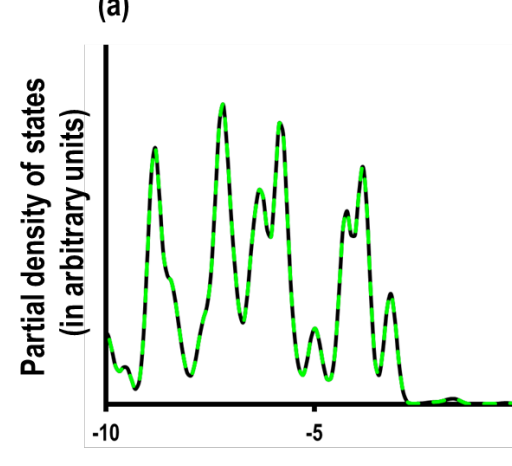

(b)

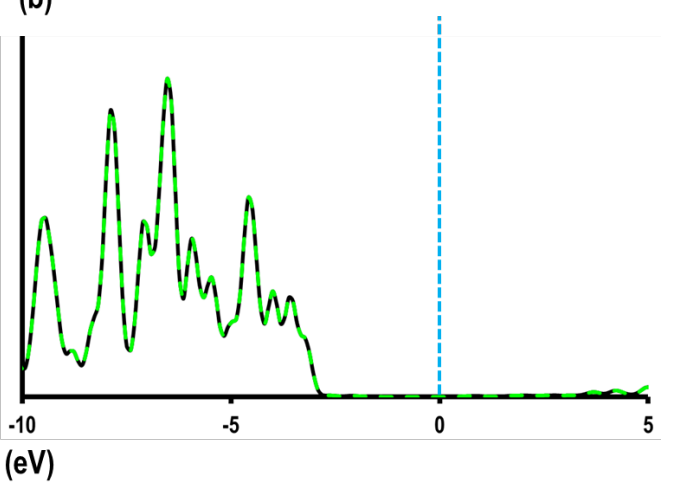

Fig. 7. Partial density of states for the $p_{z}$ orbitals of the two carbon atoms from the centre of the (a) $\operatorname{SWCNT}(6,0)$ and (b) $\operatorname{SWCNT}(9,0)$ with a length of $25 \AA$. The solid black line is the $p_{z}$ orbital signature for the first carbon atom and the green dashed line for the second carbon atom. The vertical dashed line at zero represents the Fermi energy $\left(\mathrm{E}_{\mathrm{F}}\right)$.

In Fig. 7 we explore the effect of the radius of a SWCNT on the $\mathrm{BDE}_{\mathrm{CC}}$ by considering the PDOS of the $\operatorname{SWCNT}(6,0)$ and $\operatorname{SWCNT}(9,0)$ with a similar length of $25 \AA$. The analysis on $p_{z}$ orbital in these systems show that as the radius increases the overlap between the $p_{z}$-orbitals increases significantly, which is evident from the broad overlapping signatures of SWCNT $(9,0)$ as compared to $\operatorname{SWCNT}(6,0)$. The reason for the increased overlap between the $p_{z}$-orbitals in SWCNT $(9,0)$ is related to the decrease in curvature, which is comparatively more pronounced in $\mathrm{SWCNT}(6,0)$. We conclude that the increase in $\mathrm{BDE}_{\mathrm{cc}}$ with increasing radius of $\mathrm{SWCNT}$ is related to the greater $p_{z}-p_{z}$ overlap and hence stronger chemical bonding between the carbon atoms. This is in agreement with a previous study where we have evaluated the BDE for various carbon materials (e.g. fullerene, carbon nanocones, CNTs, graphene nanoribbon) and found the BDE increases as the carbon material becomes flatter.[28,34]

\section{Conclusions}

We present a detailed DFT study of the thermodynamic properties of Single-Walled Carbon Nanotubes of various lengths, diameters and chirality. Our study shows that the BDE $\mathrm{CC}_{\mathrm{CC}}$ is inversely proportional to the curvature-squared $\left(1 / r^{2}\right)$ and inversely proportional of the SWCNT length $(1 / l)$. We derive quantitative relationships from which the BDE can be calculated as a function of size and radius of the SWCNT. This suggests that SWCNT have stronger elastic properties as their length increases due to the emergence of metallic properties and as their radius 
increases due to larger overlap of the $p$ orbitals. This clearly shows that there is a size-effect of the thermodynamic properties of SWCNTs when their length and diameter is less than 4 and 1 $\mathrm{nm}$, respectively, which should be considered in the design of materials that use SWCNT of such dimensions. Lastly, we calculate that the BDE in SWCNTs is intermediate between the BDE of a $\mathrm{C}-\mathrm{C}$ and $\mathrm{C}=\mathrm{C}$ bond, confirming partial double bond of the carbon framework.

\section{Conflicts of interest}

There are no conflicts to declare.

\section{Acknowledgments}

Via our membership of the UK's HEC Materials Chemistry Consortium, which is funded by EPSRC (EP/L000202/1), this work used the ARCHER UK National Supercomputing Service (http://www.archer.ac.uk). We acknowledge the use of Athena at HPC Midlands+, which was funded by the EPSRC (grant EP/P020232/1), in this research, via the EPSRC RAP call of spring 2018.

\section{Supplementary Material}

The coordinates of optimised SWCNT are given as supporting information (Fig. S1).

\section{References}

[1] M.S. Dresselhaus, G. Dresselhaus, R. Saito, Carbon 33 (1995) 883.

[2] M.F.L. De Volder, S.H. Tawfick, R.H. Baughman, A.J. Hart, Science 339 (2013) 535.

[3] M. Zhang, S. Fang, A. Zakhidov, S. Lee, A. Aliev, C. Williams, K. Atkinson, R. Baughman, Science 309 (2005) 1215.

[4] A.B. Dalton, S. Collins, E. Muñoz, J.M. Razal, V.H. Ebron, J.P. Ferraris, J.N. Coleman, B.G. Kim, R.H. Baughman, Nature 423 (2003) 703.

[5] K. Koziol, J. Vilatela, A. Moisala, M. Motta, P. Cunniff, M. Sennett, A. Windle, Science 318 (2007) 1892.

[6] X.J. Du, J.M. Zhang, S.F. Wang, K.W. Xu, V. Ji, Eur. Phys. J. B 72 (2009) 119.

[7] A. Pérez-Guardiola, R. Ortiz-Cano, M.E. Sandoval-Salinas, J. Fernández-Rossier, D. Casanova, A.J. Pérez-Jiménez, J.C. Sancho-García, Phys. Chem. Chem. Phys. 21 (2019) 2547.

[8] Y.V. Fedoseeva, L.L. Lapteva, A.A. Makarova, L.G. Bulusheva, A.V. Okotrub, Phys. Chem. Chem. Phys. 20 (2018) 22592. 
[9] A. Santidrián, J.M. González-Domínguez, V. Diez-Cabanes, J. Hernández-Ferrer, W.K. Maser, A.M. Benito, A. Anśon-Casaos, J. Cornil, T. Da Ros, M. Kalbáč, Phys. Chem. Chem. Phys. 21 (2019) 4063.

[10] A. Sam, V.P. K, S.P. Sathian, Phys. Chem. Chem. Phys. 21 (2019) 6566.

[11] A. Chutia, I. Hamada, M. Tokuyama, Chem. Phys. Lett. 550 (2012) 118.

[12] R. Demichelis, Y. Noël, P. D’Arco, M. Rérat, C.M. Zicovich-Wilson, R. Dovesi, J. Phys. Chem. C 115 (2011) 8876.

[13] K.Z. Milowska, J.A. Majewski, Phys. Chem. Chem. Phys. 15 (2013) 14303.

[14] G. Domínguez-Rodríguez, A. Tapia, F. Avilés, Comp. Mat. Sci. 82 (2014) 257.

[15] M. Aydin, D.L. Akins, Vib. Spectrosc. 53 (2010) 163.

[16] S.S. Han, H.M. Lee, Carbon 42 (2004) 2169.

[17] Y. Wang, A. Li, K. Wang, C. Guan, W. Deng, C. Li, X. Wang, J. Mat. Chem. 20 (2010) 6490.

[18] M. Smeu, F. Zahid, W. Ji, H. Guo, M. Jaidann, H. Abou-Rachid, J. Phys. Chem. C 115 (2011) 10985.

[19] G. Kresse, J. Furthmüller, Phys. Rev. B 54 (1996) 11169-11186.

[20] G. Kresse, J. Hafner, Phys. Rev. B 47 (1993) 558.

[21] G. Kresse, D. Jouber, Phys Rev. B 59 (1999) 1758-1775.

[22] P.E. Blöchl, Phys. Rev. B 50 (1994) 17953.

[23] J.P. Perdew, K. Burke, M. Ernzerhof, Phys. Rev. Lett. 77 (1996) 3865.

[24] H.J. Monkhorst, J.D. Pack, Phys. Rev. B 13 (1976) 5188.

[25] S. Melchor, J.A. Dobado, J. Chem. Inf. Comput. Sci. 44 (2004) 1639.

[26] , Accelrys Software Inc., Discovery Studio Modeling Environment, Release 5.5.2, San Diego: Accelrys Software Inc., 2007.

[27] S. Grimme, J. Antony, S. Ehrlich, H. Krieg, J. Chem. Phys. 132 (2010) 154104.

[28] C.D. Zeinalipour-Yazdi, E.Z. Loizidou, Carbon 146 (2019) 369.

[29] S.J. Blanksby, G.B. Ellison, Acc. Chem. Res. 36 (2003) 255.

[30] C.D. Zeinalipour-Yazdi, C. Christofides, J. Appl. Phys. 106 (2009) 054318.

[31] M.-F. Yu, O. Lourie, M.J. Dyer, K. Moloni, T.F. Kelly, R.S. Ruoff, Science 287 (2000) 637.

[32] R. Cherian, P. Mahadevan, Journal of Nanoscience and Nanotechnology 7 (2007) 1779.

[33] T. Çağin, G. Gao, W.G. III, Turk J Phys 30 (2006) 221

[34] C.D. Zeinalipour-Yazdi, E.Z. Loizidou, Carbon 115 (2017) 819. 